\title{
Evaluación del Modelo Estándar de Control Interno (MECI) y desarrollo de un modelo diferencial para el nivel territorial en Colombia*
}

\section{Internal Control Model (MECI) assessment and development of a differential model to the regions in Colombia}

Recibido: 22 de enero de 2014 Revisado: 22 de marzo de 2014 Aceptado: 27 de junio de 2014

Myrian Cubillos Benavides**

Departamento Administrativo de la Función Pública

Ileana Romea Cárdenas Manosalva****

Universidad Distrital Francisco José de Caldas

\section{RESUMEN}

La presente investigación tiene como propósito establecer las dificultades que presentan las entidades territoriales pertenecientes a los municipios de $3^{\mathrm{a}}$ a $6^{\mathrm{a}}$ categoría en la implementación del Modelo Estándar de Control Interno-MECI, teniendo en cuenta que este modelo es la herramienta que permite el desarrollo de la Políitica de Control Interno en Colombia y se constituye en el eje fundamental para el logro de los objetivos de las instituciones. Con base en el diagnóstico se determinan los aspectos para mejorar o ajustar

\footnotetext{
* Artículo de investigación.

** Correspondencia: Myriam Cubillos Benavides. Especialista en Gerencia y Gerencia Financiera. Profesional Especializado del Departamento Administrativo de la Función Pública. Correo electrónico: myriancb@yahoo.com

*** Ileana Romea Cárdenas Manosalva. Especialista en Sistemas de Gestión Ambiental. Docente de la Universidad Distrital Francisco José de Caldas. Correo electrónico: ileanaromea@gmail.com
} 
a dicho modelo, con el propósito de corregir su implementación y que a mediano plazo estas entidades puedan mejorar su gestión institucional. La investigación toma conceptos de la Nueva Gestión Pública -NGP (o New Public Management -NPM); de este enfoque se observan y analizan aspectos relacionados con el buen gobierno y la vocación por el servicio público por parte de los funcionarios del estado, los mecanismos para la democratización de la administración pública y finalmente los principios básicos sobre la responsabilidad social. La investigación es aplicada y utiliza como metodología el modelo C.I.P.P. (contexto-insumosprocesos-productos) por lo que los resultados, análisis y recomendaciones se muestran a través de sus cuatro etapas o niveles. Los principales hallazgos y conclusiones apuntan a confirmar la hipótesis planteada; se determinaron fallas en la implementación del modelo, debido a aspectos metodológicos, sobre los cuales se generan puntos de mejora 0 ajuste.

Palabras clave: control interno, gestión pública, MECI, gestión de la calidad.

\section{ABSTRACT}

This research aims to establish the difficulties presented by local authorities in the municipalities of 3rd to 6th category in implementing the Standard Model of Internal Control -MECI, considering that this model is the tool that allows the development of Internal Control Policy in Colombia and constitutes the foundation for achieving the institutions objectives. Based on the diagnosis aspects to improve or adjust this model are determined, in order to correct its implementation and in the medium term these entities can improve their corporate governance. The research takes concepts of the New Public Management (NPM); from this approach is observed and analyzed aspects related to good governance and the vocation for public service by state officials, the mechanisms for democratization of public administration and finally the basic principles of social responsibility. The research is applied and used as a model methodology CIPP (contextinput-process-products) so that the results, analysis and recommendations are shown through four stages or levels. The main findings and conclusions point to confirm the hypothesis; flaws in the implementation of the model were determined due to methodological aspects on which improvement or adjustment points are generated.

Keywords: Internal control, governance, MECI, management systems.

\section{INTRODUCCIÓN}

Los continuos cambios en la estructura general del Estado, la diversidad de las organizaciones públicas y las modificaciones normativas para el mejoramiento de la gestión pública, la eficiencia organizacional y la lucha contra la corrupción, según ley 1474 del 2011, son los referentes básicos de esta investigación.

Es necesario entonces que los modelos de gestión y control que actualmente utilizan las entidades del Estado se revisen y actualicen de acuerdo con las necesidades y retos del Estado colombiano, de modo tal que el Sistema Nacional de Control Interno realmente contribuya a mejorar la eficiencia de las organizaciones públicas en cuanto al cumplimiento de su misión y sus objetivos institucionales y para todos los órdenes y niveles de la administración pública.

En desarrollo de la política de control interno, el Estado colombiano, mediante el Decreto 1599 de 2005, adoptó el Modelo Estándar de Control Interno -MECI y obligó a su aplicación en todas las entidades 
públicas; sin embargo dada la diversidad, naturaleza y complejidad de estas instituciones, especialmente aquellas pertenecientes al nivel territorial, se han presentado dificultades en la operacionalización del modelo en cuanto a su alcance y grado de implementación; esta situación se hace evidente a través de las evaluaciones realizadas por parte del Departamento Administrativo de la Función Pública ${ }^{1}$, entidad líder de la política de Control Interno, aspecto que genera la realización de la presente investigación, la cual se orienta a la revisión y el planteamiento de cambios 0 ajustes metodológicos que mejoren o faciliten a todas las entidades del Estado colombiano la implementación total y efectiva del MECI. La situación más difícil para la implementación del MECI se presenta en los municipios que según su realidad presupuestal y poblacional se clasifican como de $3^{\mathrm{a}}$ a $6^{\mathrm{a}}$ categoría (menos de 30.000 habitantes y con ingresos de hasta $50.000 \mathrm{smlmv})$.

En este contexto se plantea la siguiente hipótesis: Los municipios de $3^{\mathrm{a}}$ a $6^{\mathrm{a}}$ categoría requieren de una metodología específica para la implementación del Modelo Estándar de Control Interno -MECI.

Se plantea como propósito principal formular ajustes metodológicos a la implementación del modelo estándar de control interno, de modo que se constituya en una herramienta diferencial que facilite su aplicación por todas las entidades públicas pertenecientes a los municipios de $3^{\mathrm{a}}$ a $6^{\mathrm{a}}$ categoría.

1 DAFP. Informe Seguimiento al cumplimiento del Decreto 3181 de 2009 Sistema de Control Interno. Septiembre 2010.

DAFP. Informe Ejecutivo Anual Sistema de Control Interno vigencia 2008. Mayo 2009.

DAFP. Informe Ejecutivo Anual Sistema de Control Interno vigencia. Mayo 2010.

DAFP. Informe Seguimiento al cumplimiento del Decreto 3181 de 2009 Sistema de Control Interno. Septiembre 2010.
Debido a la condición de entidades públicas de la población objeto estudio, el desarrollo de la investigación se sustentó en el marco constitucional y legal, por ello se planteó el marco teórico bajo tres conceptos o líneas de acción a saber:

- La Nueva Gestión Pública -NGP y el Sistema de Control Interno en Colombia: En esta línea teórica se abordan los principales retos para los gobiernos una vez fueron surtidos los lineamientos de tipo económico que llevaron a los países a disminuir su aparato estatal, derivados principalmente de las formulaciones del denominado Consenso de Washington (Serrano, 2000) y su posterior cambio de rumbo, orientados a la ampliación de la capacidad institucional, cuyas bases han sido desarrolladas por el profesor Michael Barzelay a partir de un estudio en el que analiza acciones de distintos programas gubernamentales implementados en el Estado de Minnesota (EE.UU.), en lo que se planteó como el "Paradigma posburocrático" a partir del cual se estipula:

En este paradigma el control coercitivo debe dar paso a un apego a las normas desde su comprensión e interiorización (autocontrol) [...] También se pretende dar paso a la identificación misional, de servicios, clientes y resultados, más que la mera especificación de funciones, autoridades y estructuras. (Barzelay, 2003, p. 135)

A partir de estos conceptos, la denominada Nueva Gestión Pública o Nerw Public Management-NPM entrega de manera general "una visión más económica y gerencial de la administración gubernamental, haciendo énfasis en la ampliación de la capacidad institucional y en la eficacia de la acción estatal para el buen funcionamiento de los mercados" (López, 2003).

En concordancia con tales cambios, el Plan Nacional de Desarrollo 2010-2014 "Prosperidad para todos" en 
su capítulo VII, Soportes transversales de la prosperidad democrática, plantea que "el mejoramiento continuo de la Administración Pública como eje fundamental y estratégico para consolidar el Buen Gobierno, se basará en el fortalecimiento de herramientas de gestión de gran impacto que se consoliden en un sistema integrado, permitiendo el buen uso de los recursos y la capacidad del Estado para producir resultados en pro de la satisfacción de los intereses ciudadanos" (2011, p. 638), por lo que el modelo MECI se constituye en una de dichas herramientas y su mejora contribuye a tales propósitos.

- Buen gobierno y vocación de servicio: Esta línea teórica representa un eje fundamental para el fortalecimiento de las capacidades institucionales, el mejoramiento de la eficiencia y efectividad de las organizaciones públicas, teniendo en cuenta que con el compromiso y competencias de los funcionarios es posible alcanzar los objetivos de las instituciones. Este eje promueve rasgos esenciales en el servidor público como son: Probidad, Responsabilidad, Transparencia, Imparcialidad, Eficiencia y Disponibilidad a la respuesta oportuna, los cuales se indagan a través del componte ambiente de control del modelo MECI.

- Democratización de la administración pública: Esta línea conceptual se analiza a través de los siguientes lineamientos básicos:

La participación ciudadana y el control social a la gestión pública corresponden a la concepción de un nuevo Estado, que permite y apoya la intervención de los ciudadanos en la gestión pública. Esta intervención busca que los ciudadanos participen vigilando la gestión del Estado y que cooperen prepositivamente para el mejoramiento de las funciones públicas y el uso de los recursos. En consecuencia todas las entidades y organismos tienen la obligación de desarrollar su gestión en consonancia con los principios de democracia participativa y democratización de la administración pública. (Documento Plan Nacional de Formación para el Control Social a la Gestión Pública, 2004-2007)

Este concepto es esencial como punto de contacto vital con los usuarios o ciudadanos y alrededor del cual la prestación de servicios a éstos puede ser mejorada constantemente, aspectos indagados de manera amplia en los componentes Información y Comunicación Pública del modelo MECI.

De acuerdo con estos conceptos y a partir del desarrollo de tres mapas conceptuales relacionados con estas tres líneas de acción, las investigadoras centraron el análisis en tres enfoques teóricos que se definen a continuación:

a) Direccionamiento Estratégico: busca determinar la alineación con el gobierno nacional y departamental, así como la articulación institucional con la participación de los grupos de interés, partiendo de una base ética y de cultura organizacional promovida por sus dirigentes.

b) Gestión Administrativa y Gerencial: uso y manejo de herramientas de gestión, permitiendo el buen uso de los recursos, se incluye el uso y manejo de herramientas tecnológicas -TIC's- en pro de la satisfacción de los intereses ciudadanos.

c) Mejoramiento Continuo: implementación de modelos de gestión de la calidad u otros que permitan mejorar la prestación del servicio.

Estos tres enfoques se asociaron con los componentes del modelo que se considera que apunta directamente 0 aporta a su cumplimiento por parte de las entidades del Estado, teniendo en cuenta asimismo su relevancia y los requerimientos que cada elemento del modelo al interior desarrolla, estableciendo la siguiente tabla de asignación de pesos (ver cuadro 1): 
Cuadro 1. Asignación de pesos por enfoque

\begin{tabular}{|c|c|c|c|}
\hline $\begin{array}{l}\text { ENFOQUES TEÓRICOS PARA } \\
\text { DETERMINAR LAS PREGUNTAS }\end{array}$ & $\begin{array}{l}\text { PESO POR } \\
\text { ENFOQUE }\end{array}$ & $\begin{array}{l}\text { COMPONENTE } \\
\text { DEL MECI }\end{array}$ & $\begin{array}{c}\text { PESO POR } \\
\text { COMPONENTE }\end{array}$ \\
\hline \multirow{3}{*}{$\begin{array}{l}\text { "Direccionamiento Estraté́gico } \\
\text { (Busca determinar la alineación con el gobierno } \\
\text { nacional y departamental, así como la articulación } \\
\text { institucional con la participación de los grupos de } \\
\text { interés, partiendo de una base ética y de cultura } \\
\text { organizacional promovida por sus dirigentes)" }\end{array}$} & \multirow{3}{*}{$60 \%$} & Direccionamiento Estratégico & $30 \%$ \\
\hline & & Información (Primaria) & $10 \%$ \\
\hline & & Ambiente de control & $20 \%$ \\
\hline \multirow{4}{*}{$\begin{array}{l}\text { "Gestión Administrativa y Gerencial } \\
\text { (Uso y manejo de herramientas de gestión, } \\
\text { permitiendo el buen uso de los recursos, } \\
\text { se incluye el uso y manejo de herramientas } \\
\text { tecnológicas -TIC's- en pro de la satisfacción de } \\
\text { los intereses ciudadanos.)" }\end{array}$} & \multirow{4}{*}{$30 \%$} & $\begin{array}{l}\text { Información (Secundaria + Sistemas de } \\
\text { Información) }\end{array}$ & $5 \%$ \\
\hline & & Actividades de Control & $8 \%$ \\
\hline & & Comunicación Pública & $7 \%$ \\
\hline & & Administración del Riesgo & $10 \%$ \\
\hline \multirow{3}{*}{$\begin{array}{l}\text { "Mejoramiento Continuo } \\
\text { (Implementación de modelos de gestión de } \\
\text { la calidad u otros que permitan mejorar la } \\
\text { prestación del servicio)" }\end{array}$} & \multirow{3}{*}{$10 \%$} & Evaluación Independiente & $2 \%$ \\
\hline & & Autoevaluación & $3 \%$ \\
\hline & & Planes de Mejoramiento & $5 \%$ \\
\hline TOTAL & $100 \%$ & & $100 \%$ \\
\hline
\end{tabular}

Fuente: elaboración propia.

Para la estructura de asignación de pesos por enfoque que se observa en el Cuadro 1, se realizó una adaptación partiendo de los cinco ejes del Plan Nacional de Desarrollo 2010-2014 "Prosperidad para todos", denominados ejes de "Buen Gobierno", los cuales tienen como "fin último velar porque la visión y prioridades de las entidades del Estado cuenten con planes, indicadores de gestión y mecanismos de coordinación, que permitan una rendición de cuentas permanente y transparente a los ciudadanos" (2011, p. 628), y la experticia de funcionarios consultados dentro del Departamento Administrativo de la Función Pública, quienes dentro de la evaluación al modelo (MECI) para la vigencia 2012 adoptaron una serie de pesos sobre estos mismos cinco ejes de buen gobierno, los cuales pueden ser consultados en el Informe Ejecutivo Anual de Control Interno vigencia 2012.
Con estas bases teóricas y los resultados de la encuesta, las conclusiones y recomendaciones se orientan a determinar cuáles aspectos o elementos metodológicos del modelo deben ser ajustados o fortalecidos.

\section{METODOLOGÍA}

La investigación parte de un enfoque positivista el cual plantea que una proposición o enunciado tiene sentido solo si resulta verificable por medio de la experiencia y la observación, en tal sentido el tipo de investigación es aplicada en el entendido que con los resultados que se presentan es posible coadyuvar a la solución de los actuales problemas frente al desarrollo del modelo MECI. En concordancia con tales principios se hace 
uso del modelo C.I.P.P. (contexto-insumos-procesosproductos) y se desarrollaron las cuatro etapas o niveles determinados en dicho modelo.

El grupo objeto de estudio establecido fueron las entidades pertenecientes a los municipios de $3^{\mathrm{a}}$ a $6^{\mathrm{a}}$ categoría, contemplados en el artículo $6^{\circ}$ de la Ley 617 de 2000, determinadas como las que directamente muestran las debilidades ya mencionadas frente a la implementación del modelo. El universo de entidades de acuerdo a este grupo objetivo, así como aquellas que fueron seleccionadas para responder la encuesta, se establecieron sobre la base de datos suministrada por el Departamento Administrativo de la Función Pública (DAFP), teniendo en cuenta el diseño muestral desarrollado.

El modelo C.I.P.P. (contexto-insumos-procesosproductos) ya mencionado, bajo un enfoque analíticoracionalista, determina que la evaluación es el proceso de identificar, obtener y proporcionar información útil y descriptiva acerca del valor y el mérito de las metas, la planificación, la realización y el impacto de un objeto determinado, con el fin de servir de guía para la toma de decisiones, solucionar problemas de responsabilidad y promover la comprensión de los fenómenos implicados (Correa, Zapata y Restrepo, 1996).

Con base en lo anterior a continuación se muestra el alcance de cada una de las etapas:

Para la primera etapa denominada "delineación", se determinaron los objetivos de la investigación y la hipótesis relacionados en la introducción del presente documento. En esta etapa se estructuraron las encuestas tomando como referencia los resultados de los mapas conceptuales bajo los tres enfoques teóricos, construidos a partir del Marco Teórico y los criterios

2 Modelo desarrollado por el profesor Daniel Leroy Stufflebeam Ph.D de la Universidad Western Michigan en Estados Unidos. para el enfoque de la encuesta. Con estos fundamentos, producto del desarrollo del marco teórico de la investigación, se enlazaron los diferentes componentes del Modelo Estándar de Control Interno -MECI y se diseña el cuestionario para la recolección de la información. Para la validación de este cuestionario se llevó a cabo una prueba piloto con siete entidades territoriales de municipios ubicados en Cundinamarca, lo que permitió realizar los ajustes correspondientes a las preguntas y determinar la forma de aplicación del cuestionario.

Para la segunda etapa denominada "obtención de la información”, se realizó el diseño muestral, partiendo del universo o población objetivo, que en este caso corresponde al ámbito de aplicación de la Ley 87 de 1993, donde establece a todos los organismos y entidades de las ramas del poder público en sus diferentes órganos y niveles. A partir de éste universo, en la clasificación por categorías de municipios establecidos en la Ley 617 de 2000, artículo $6^{\circ}$, se toman las entidades pertenecientes al orden Territorial en las categorías $3^{\mathrm{a}}$ a 6 registradas en la base de datos del Departamento Administrativo de la Función Pública, estableciendo un universo para la investigación de 3681 entidades (DAFP, 5 de agosto de 2013).

Para la determinación del tamaño de la muestra, mediante el uso del método probabilístico y haciendo uso del muestreo aleatorio estratificado, se encuentra que el número de entidades a encuestar es de 116.

Una vez obtenido el cálculo de entidades, se procedió a codificar la lista de entidades y haciendo uso de la herramienta complementos estadísticos del programa Excel se determinaron de forma aleatoria las instituciones a contactar para el diligenciamiento de la encuesta.

A partir de esta selección se generaron las correspondientes listas de distribución para la aplicación del instrumento, para este efecto se utilizó un recurso web 
que permitió recolectar la información en línea a través de la página www.encuestafacil.com, la cual mediante suscripción permitió acceder a varios recursos como la estructuración de las preguntas con los condicionamientos respectivos, alojar la información recolectada y generar los resultados consolidados que se mostrarán más adelante. Es importante precisar que la decisión de utilizar este medio respondió a las observaciones recibidas por parte de la entidades que participaron en la prueba piloto realizada para validar la encuesta, ya que las indicaciones para ir avanzando hacia aquellas preguntas que dependían de respuestas positivas iniciales, generaba confusión en el diligenciamiento.

Asimismo, en esta etapa se realizó el análisis estadístico de la información por cada uno de los subsistemas del MECI indagados en la encuesta.

Para la tercera etapa denominada "análisis de la información", se tuvieron en cuenta los tres enfoques teóricos determinados: Direccionamiento Estratégico, Gestión Administrativa y Gerencial y Mejoramiento Continuo, producto del marco teórico de la investigación, a partir de los cuales se pudieron establecer los elementos técnicos del modelo que de acuerdo a la asignación de pesos y los resultados de las preguntas, son los de mayor debilidad o dificultad para su desarrollo en las entidades objeto de estudio en cada uno de los subsistemas, componentes y elementos del MECI.

Finalmente para la cuarta etapa denominada "aplicación de la información", se establece que es procedente suministrar al Departamento Administrativo de la Función Pública los resultados de la investigación, para que de acuerdo con las recomendaciones generadas, dicha institución defina los mecanismos para ajustar el modelo y establezca las estrategias para la implementación del nuevo modelo en las entidades del orden territorial.

Con la investigación se cubrieron 22 de los 32 departamentos del país, que corresponde a un $69 \%$ y en total se recibieron 122 cuestionarios diligenciados.

Del total de entidades encuestadas, el $34 \%$ correspondió a alcaldías municipales, $21 \%$ a empresas sociales del Estado (hospitales del municipio), $18 \%$ a concejos municipales, $12 \%$ a personerías municipales, $7 \%$ a empresas de servicios públicos del municipio, $4 \%$ a entidades descentralizadas del municipio, $2 \%$ a contralorías municipales y el restante porcentaje corresponde a una Gobernación y una empresa industrial y comercial del estado.

De forma general, es posible establecer con estos datos básicos sobre tipos de entidades y funcionarios que laboran en las áreas de control interno, que en una porcentaje superior al $60 \%$ las entidades no cuentan con un Jefe de Control Interno y que las personas que ejercen tales funciones son personas que desempeñan cargos en procesos misionales, es decir, que desarrollan funciones en cargos de planta de la entidad relacionados con las actividades propias de la gestión y operación de la entidad, lo que necesariamente afecta de manera importante la objetividad y desempeño del funcionario frente al tema de control interno, afectando por ende la aplicación del modelo y la conciencia que deben tener los funcionarios sobre la responsabilidad en la aplicación de los métodos y procedimientos de control interno que se encuentran a lo largo de la organización.

\section{RESULTADOS Y DISCUSIÓN}

De acuerdo con los enfoques teóricos explicados en la introducción y posteriormente ampliados en la metodología del presente artículo, a saber: Direccionamiento 
Estratégico, Gestión Administrativa y Gerencial y Mejoramiento Continuo, los resultados en las entidades objeto de estudio son los siguientes:

\section{Enfoque Direccionamiento Estratégico}

Este enfoque tiene un peso de $60 \%$ basado en el análisis del marco teórico y contiene los componentes del MECI: direccionamiento estratégico, ambiente de control e información primaria. Por lo tanto, de acuerdo a los resultados de la encuesta, a continuación se muestran los análisis detallados:

\section{Componente direccionamiento estratégico}

Con un peso de $30 \%$ dentro del enfoque, de forma general para este componente es posible afirmar que las entidades sí establecen una planeación institucional basada en análisis del entorno, que han estructurado sus procesos y respectivos mapas, sin embargo la mayor debilidad se encuentra en la articulación entre los procesos que componen dicho mapa y los planes y programas que desarrollan, se podría inferir en este caso que si bien se han caracterizado los procesos, se sigue trabajando por áreas funcionales, lo que no permite su articulación efectiva.

\section{Componente información primaria}

Con un peso de $10 \%$ dentro del enfoque, sobre este componente es posible analizar que específicamente para el manejo de información proveniente de los usuarios o ciudadanos, la cual debe ser recolectada, procesadas y analizada para mejorar los procesos de gestión y atención a los mismos, la mayoría de entidades manifiesta contar con los mecanismos necesarios, sin embargo, un porcentaje importante (25\%) no la utiliza para estos fines, por lo que es importante reforzar este aspecto para que dicha información sea utilizada para la mejora en la prestación de los servicios a los ciudadanos.

\section{Componente ambiente de control}

Con un peso del $20 \%$ dentro del enfoque, de forma general se puede afirmar que si bien el tema de clima organizacional y su respectiva evaluación se encuentran establecidas en las entidades, las acciones que se toman de forma posterior están relacionadas únicamente con actividades de capacitación en su mayoría, sin tener en cuenta actividades que propendan por el desarrollo y mejora de las competencias de los funcionarios.

Por otro lado, el código de ética y el código de buen gobierno existen en las entidades, pero se evidencian vacíos en el conocimiento del tema y de las metodologías que para su construcción existen. En consecuencia, estos aspectos metodológicos deben ser un punto esencial para la mejora del modelo, debido a la importancia que representan como elementos básicos necesarios para generar un ambiente favorable de control.

\section{Enfoque Gestión Administrativa y Gerencial}

Este enfoque tiene un peso de $30 \%$ basado en el análisis del marco teórico y contiene los componentes del MECI: información secundaria, sistemas de información, actividades de control, comunicación pública y administración del riesgo. Por lo tanto de acuerdo a los resultados de la encuesta, a continuación se muestran los análisis detallados:

\section{Componentes información secundaria y sistemas de información}

Con un peso de $5 \%$ dentro del enfoque, para este componente de forma general se puede analizar que el tema de mayor dificultad tiene que ver con el manejo de la ley de archivo, aspecto muy relevante tratándose de un mecanismo que debe facilitar la preservación 
de toda la memoria institucional, cuya debilidad abre espacios para posibles actos de corrupción al no contar con registros confiables de la gestión. Por lo que este aspecto tendría que configurarse con mayor profundidad en el modelo, atendiendo los lineamientos del Archivo General de la Nación.

Por otro lado en cuanto a los sistemas de información implementados, las entidades tienden a identificarlos únicamente con el software de registros contables u otros externos donde reportan información, pero no cuentan con software de captura de información o de generación de reportes de indicadores u otras herramientas gerenciales para el manejo de información de la gestión, razón por la cual podría cuestionarse la afirmación que tales mecanismos facilitan la entrega de información a la ciudadanía en forma clara y comprensible. Estos aspectos tendrían que puntualizarse para que sean entendidos correctamente y que puedan ir avanzando en su implementación.

\section{Componente actividades de control}

Con un peso del $8 \%$ dentro del enfoque, en forma general para el componente se puede afirmar que si bien los procedimientos y los indicadores de gestión son conocidos por la mayoría de funcionarios, en un porcentaje cercano al $37 \%$ los desconoce o la entidad no las ha establecido para su aplicación, igual análisis se desprende para el manual de operaciones, por lo que es necesario reforzar estos temas, sobre todo en su contenido metodológico.

\section{Componente comunicación pública}

Con un peso de $7 \%$ dentro del enfoque, de forma general para este componente las entidades reconocen las principales políticas que desarrollan los mecanismos de participación ciudadana, así como los lineamientos de la ley anti-trámites, por lo que es posible establecer que son elementos con gran desarrollo y fortaleza en su aplicación.

\section{Componente administración del riesgo}

Con un peso del $10 \%$ dentro del enfoque, sobre este componente se puede concluir que existe un desconocimiento del tema en un porcentaje importante de entidades (30\%), para aquellas entidades que sí conocen el tema, la metodología a la que más se acude es a la guía del DAFP, de éstas aunque la conocen no la han aplicado en un porcentaje del $18 \%$. Asimismo la participación para su construcción es otro factor a tener en cuenta, ya que en un $45 \%$ el proceso para su elaboración está delegado o no se ha realizado, por lo que es preciso seguir profundizando en el tema dentro del modelo.

\section{Enfoque Mejoramiento Continuo}

Este enfoque tiene un peso de $10 \%$ basado en el análisis del marco teórico y contiene los componentes del MECI: autoevaluación, evaluación independiente y planes de mejoramiento. Por lo tanto de acuerdo a los resultados de la encuesta, a continuación se muestran los análisis detallados:

\section{Componente autoevaluación}

Con un peso del $3 \%$ dentro del enfoque, en general es posible establecer que existe un desconocimiento del tema de autoevaluación y sus herramientas, por lo que se hace necesario reforzar este componente dentro del modelo y especificar las herramientas que existen para realizarla.

\section{Componente evaluación independiente}

Con un peso de $2 \%$ dentro del enfoque, en general para este componente se identifican las principales dificultades en la caracterización de las oficinas de Control Interno, que en su mayoría cuentan con un 
solo funcionario o realizan contrataciones por orden de prestación de servicios para la ejecución de tales funciones, actividad que legalmente es irregular, ya que solamente es posible realizar contratación con empresas de reconocida experiencia y trayectoria, sin embargo por desconocimiento, las entidades las siguen realizando para suplir las funciones de control interno. Igual situación e incluso más compleja es el de aquellas entidades donde funcionarios con cargos en procesos misionales son quienes llevan a cabo actividades de auditoría. Por lo tanto será preciso generar lineamientos claros al respecto para minimizar el impacto de estas realidades.

\section{Componente planes de mejoramiento}

Con un peso del $5 \%$ dentro del enfoque, en general para este componente las principales debilidades se ubican, por un lado, en el levantamiento de los planes de mejoramiento y el respectivo seguimiento por parte de los líderes de proceso, y posteriormente por parte del Jefe de Control Interno o quien hace sus veces, situación que dificulta que este elemento realmente redunde en el mejoramiento de la gestión.

Por otro lado los planes de mejoramiento individual tienden a relacionarse únicamente con capacitaciones e incentivos, que si bien ayudan a la mejora individual en algunos aspectos, no tocan de manera específica las competencias laborales de los funcionarios.

Por lo anterior ambos aspectos tendrán que precisarse e incluir herramientas para su formal aplicación.

En consecuencia, y teniendo como base los pesos por enfoque y los resultados de la encuesta en cada uno de sus respectivos componentes es posible concluir que los de menor desarrollo son los siguientes:

- Información primaria y sistemas de información

- Administración del riesgo

- Autoevaluación
- Evaluación independiente

- Planes de mejoramiento

Ahora bien, los componentes que requieren un fortalecimiento en cuanto a sus metodologías de aplicación son:

- Información primaria

- Ambiente de control

- Actividades de control

Finalmente es posible resaltar que los componentes de mayor desarrollo son:

- Direccionamiento estratégico

- Comunicación pública

\section{CONCLUSIONES Y RECOMENDACIONES}

Una vez realizado el análisis de los componentes del Modelo Estándar de Control Interno -MECI y definidas sus relaciones con los planteamientos del marco teórico se encuentra que el enfoque de operación por procesos se constituye en la estructura central del modelo, dado que contiene el diseño de los procesos a través de los cuales se articula toda la planeación institucional y las entidades en general manifiestan que lo tienen desarrollado; por lo tanto es necesario precisar los puntos de articulación entre el modelo de procesos y la planeación, para que las caracterizaciones de proceso sean el punto de partida para el desarrollo de metodologías como la de administración del riesgo o la definición de los indicadores de gestión, que como se ha venido analizando en los resultados de la encuesta, son los de mayor dificultad para su implantación efectiva.

En cuanto a los mecanismos de recolección de información abiertos a la ciudadanía, si bien se encuentra 
que las entidades los han diseñado e implantado, es necesario reforzar la importancia de realizar el análisis de la información recolectada, con el fin de que se convierta en un insumo para la mejora en la prestación de los servicios a los ciudadanos, de modo tal que los ciudadanos reconozcan no solamente que son escuchados, sino que sus opiniones son tenidas en cuenta y que la entidad aplica los correctivos necesarios para mejorar la prestación de sus servicios.

Dentro de los elementos indagados en el ambiente de control, es necesario incluir metodologías para la construcción del código de ética y el código de buen gobierno, teniendo en cuenta que las entidades los tienen desarrollados como documentos para dar cumplimiento a una exigencia legal, pero no se usan o no son entendidos como elementos que permitan reforzar el comportamiento ético de los funcionarios. Asimismo es necesario mencionar que, al ser el factor humano determinante en toda institución para el normal desarrollo de su gestión, las metodologías y conceptos relacionados con el clima laboral y las acciones para su mejora deben incluirse como parte de la agenda de los directivos, ya que es posible analizar que existe desconocimiento del tema, al reducir las acciones frente al recurso humano a procesos de capacitación, sin incluir el tema de desarrollo de capacidades y competencias de los funcionarios.

Frente al componente de información secundaria es preciso incluir como anexo técnico dentro del manual que genera los lineamientos del modelo, las guías y procedimientos relacionados con la Ley general de archivo, ya que si bien el Archivo General de la Nación como líder de esta política los viene formulando, la mayoría de entidades desconocen el tema y no aplican sus lineamientos.

Sobre las principales actividades de control como son el diseño y aplicación de indicadores de gestión, procedimientos y el manual de operaciones, el refuerzo debe ser de tipo metodológico, ya que los elementos se conocen y en general están implementados pero no hacen parte integral de la gestión, es decir se cuenta con indicadores pero no se alimentan, por lo que en la práctica no existe información sobre los resultados reales de la gestión y el cumplimiento de la planeación institucional.

Para el componente de administración del riesgo, si bien las metodologías existentes para el proceso de gestión del riesgo son conocidas por las entidades, especialmente la emitida por el Departamento Administrativo de la Función Pública -DAFP, aún se presentan dificultades para su implantación principalmente por la falta de participación de quienes ejecutan los procesos en su construcción y de los responsables por su posterior seguimiento; se tiende principalmente a delegar en quienes ejercen las funciones de control interno su desarrollo, por lo que este elemento requiere de un mayor énfasis en la necesidad de participación de líderes de proceso y directivos, asunto que podría incluirse en las estrategias de difusión del DAFP.

Entre los componentes analizados para el enfoque mejora continua, el de mayor dificultad para su implantación es el de Autoevaluación, ya que en la mayoría de entidades no se conoce ni se practica; no se conocen metodologías para su aplicación, por lo cual es recomendable generar una guía que desarrolle el tema y plantee las respectivas metodologías, de modo tal que se encuentren formas prácticas para iniciar su implementación. Cabe resaltar en este punto que los procesos de autoevaluación en las entidades son los que permiten sentar las bases para generar una cultura del autocontrol, para que los procesos de seguimiento estricto relacionados con las auditorías de los entes de control y las realizadas por la oficina de control interno puedan ser asumidas con mayor capacidad, generando 
resultados favorables para la entidad, de allí la importancia de su fortalecimiento.

Relacionado con lo anterior, en los planes de mejoramiento que surgen tanto de las auditorías realizadas por los entes de control, como de las de gestión realizadas por la oficina de control interno o quien haga sus veces en las entidades, se presentan dificultades principalmente debido al desconocimiento de metodologías para su elaboración; se encuentra que se levantan acciones que no corresponden al hallazgo u observación presenciada y en consecuencia no se puede hacer el seguimiento correspondiente, debido a que no hay coherencia entre el hallazgo y la acción para su mejora. Esta falta de conocimiento de quien elabora el plan, determina que la oficina de control interno no pueda realizar los cierres correspondientes, por lo que vigencia tras vigencia quedan planes de mejoramiento abiertos que no generan valor ni aportan para la mejora continua de los procesos. En consecuencia es relevante que se estructure una guía metodológica sobre el tema, con aspectos básicos que permitan levantar acciones coherentes con las observaciones encontradas.

Finalmente, como resultado del análisis de caracterización de las oficinas de control interno, se encuentra que en su mayoría cuentan con un solo funcionario o que la labor de control interno se contrata por orden de prestación de servicios; es importante que el DAFP, como líder de la política de control interno, formule lineamientos claros al respecto, de modo que se puedan establecer perfiles para quienes desarrollan o tienen asignadas las funciones de control interno, con el fin de mitigar el riesgo sobre aquellos funcionarios que simultáneamente desarrollan actividades misionales y deben realizar procesos auditores, actividad completamente contraría a la filosofía del control interno y de la práctica profesional de auditoría interna. 


\section{REFERENCIAS}

Cochran, W. (1971) Técnicas de Muestreo. Primera Edición (Cáp. 5, p.127). Mexico: Editorial CECSA.

Colombia. Congreso de la República. Ley 87 (29, noviembre, 1993). Diario Oficial. Bogotá D.C., 1993. No. 41.120.

Colombia. Congreso de la República. Ley 617 (06, octubre, 2000). Diario Oficial. Bogotá D.C., 1993. No. 44.188

Colombia. Congreso de la República. Ley 1474 (12, julio, 2011). Diario Oficial. Bogotá D.C., 2011. No. 48.128

Colombia. Congreso de la República. Decreto 1599 (20, mayo, 2005). Diario Oficial. Bogotá D.C., 2005. No. 45.920

Correa, S., Zapata, A. y Restrepo, B. (1996). Investigación Evaluativa. Instituto Colombiano para el Fomento de la Educación Superior, ICFES. Bogotá. Módulos de Investigación Social.

Departamento Administrativo de la Función PúblicaDAFP. (septiembre, 2010). Informe Seguimiento al cumplimiento del Decreto 3181 de 2009 Sistema de Control Interno.
Departamento Administrativo de la Función Pública -DAFP. (05 de agosto de 2013). Aplicativo MECI-Calidad.

Departamento Administrativo de la Función Pública -DAFP. Documento Plan Nacional de Formación para el Control Social a la Gestión Pública. Bogotá 2004-2007.

Departamento Nacional de Planeación. (2011). Plan Nacional de Desarrollo 2010-2014: Prosperidad para todos. Bogotá.

González, A. (s. f.) "Organismos ad-hoc de lucha contra la corrupción y códigos de ética". En "Ética Y Administración Pública", Documentos del foro Iberoamericano de jefes de Estado y de Gobierno, Comisión Presidencial para la Reforma al Estado COPRE. Caracas Venezuela.

Lafuente, A. y Viñuales V. (2003). Responsabilidad Social Corporativa y Políticas Públicas. Documento de trabajo. Madrid España.

López, A. (2003). La Nueva Gestión Pública: Algunas Precisiones para su Abordaje Conceptual. Instituto Nacional de La Administración Pública- Dirección de Estudios e Información -INAP.

Serrano, J. F. (2000). El "Consenso de Washington" ¿Paradigma económico del capitalismo triunfante? Recuperado de http://www.eclac.cl/Mujer/proyectos/gobernabilidad/manual/mod01/13.pdf 